\title{
(1)Psicogente
}

\section{Abordaje de las urgencias psiquiátricas en los hospitales generales: una propuesta desde el marco de las urgencias subjetivas}

\section{Approaching the emergency psychiatric services in hospitals: a proposal based on subjective emergency}

ISSN 0124-0137
EISNN 2027-212X EISNN 2027-212X
Artículo de Revisión

Copyright 2018 by Psicogente

\section{Correspondencia de autores:}

nathalyr18@gmail.com

angelagomezocampo@gmail.com

Recibido: $10-12-16$

Aceptado: 09-05-17

Publicado: 01-01-18

\author{
Nathaly Rivera Romero \\ Fundación Universitaria de Ciencias de la Salud, FUCS, Bogotá, Colombia \\ Ángela Patricia Gómez Ocampo \\ I.E. San Antonio, Rionegro, Colombia
}

Resumen

Objetivo: El siguiente artículo de revisión bibliográfica busca responder por la intervención en salud mental o la atención al paciente psiquiátrico en los servicios de urgencias, con la finalidad de ampliar los términos de la intervención en psiquiatría con los desarrollos que al respecto ha realizado el psicoanálisis apoyándose en el concepto psicoanalítico de urgencias subjetivas, lo cual podría plantearse como una intervención de la urgencia psiquiátrica centrada en el sujeto

Método: Se realizó una revisión de la literatura de tipo descriptivo, desarrollada a través de un proceso de búsqueda de información, analizando documentos existentes en el metabuscador de Google Académico y en las bases de datos: Ebsco Host, PubMed, Scielo, Redalyc, Cuiden, y BVS, los textos fueron elegidos por pertinencia, relevancia y accesibilidad, tomando como referencia los avances teóricos de los investigadores Belaga, Sotelo y Seldes

Resultados: Encontrando que la demanda de atención psiquiátrica urgente está tomando cada vez mayor fuerza, especialmente en los hospitales generales, por lo cual es indispensable que el personal de los servicios de urgencias tenga una formación que responda a estas exigencias, situando que tanto los manuales diagnósticos y la psicofarmacología, como la palabra, son herramientas de las que disponen los equipos asistenciales en las instituciones hospitalarias.

Conclusiones: Realizando en el abordaje de la urgencia psiquiátrica un análisis clínico que trascienda la presentación superficial de los síntomas y tenga en cuenta al sujeto del inconsciente, reconociendo que la superación de la crisis por medio de una intervención del cuerpo biológico no es suficiente.

Palabras Claves:

Urgencia psiquiátrica, Urgencias subjetivas, Hospital general, Interfase.

Abstract

Objective: This paper is a response to mental health interventions and psychiatric patient care in the Emergency Psychiatric Services, which pose an urgent psychiatric intervention focused on the patient, in order to expand the terms of intervention in Psychiatry, conducted by psychoanalysis' related to the psychoanalytical concept "subjective emergency".

Method: For this research, a descriptive literature review was carried out, developed through a process of data searching and analyses of existing documents in Google Scholar meta-search engine and databases such as: Ebsco, Host, PubMed, Scielo, Redalyc, cuiden and BVS. The texts were chosen based on relevance and accessibility, taking into account the theoretical progress of the researchers Belaga, Sotelo and Seldes

Results: Taking into account that urgent psychiatric care demands are increasing, especially in hospitals, it is essential that emergency services staff have training that responds to these demands; placing both diagnostic manuals and psychopharmacology as available tools for health care teams in health institutions.

Conclusion: This training performs a clinical analysis that goes beyond the superficial presentation of symptoms and keeping in mind the patient in the approach to psychiatric emergency, recognizing that the overcoming the crisis by means of a intervention from the biological body is not enough.

Key words:

Psicoge Psychiatric urgency, Subjective urgencies, General hospital, Interface 


\section{INTRODUCCIÓN}

La intervención en salud mental representa un reto para los profesionales que de una u otra forma tienen que ver con la atención a pacientes con afecciones catalogadas -según los manuales de diagnóstico- como trastornos mentales (Bulbena, Martin \& Arcega, 2007, p.2). En particular, los trastornos muestran una gran variedad de presentaciones que se inscriben dentro de lo que se ha denominado multicausalidad de la enfermedad mental en el modelo biopsicosocial (ArangoDávila, Rojas \& Moreno, 2008, p.539; Chinchilla, 2010, p.6; Moya, Piñera \& Mariné, 2011, p.6; Berenzon \& Vargas, 2012, p.12) y según la Organización Mundial de la Salud -OMS- la demanda de atención en salud mental en el mundo es significativamente mayor comparada con la capacidad de respuesta de las instituciones (WHO, 2011). Así, al tener en cuenta el estado global de la salud mental y los estudios realizados en Colombia, se puede probar lo pertinente que resulta ocuparse de formular propuestas de intervención que contribuyan a avanzar en esta vía.

Arango-Dávila et al. (2008) han resaltado que Colombia cuenta con una problemática de salud mental y psiquiatría que supera la situación de los países bolivarianos y centroamericanos, además de ocupar entre cinco grupos de patologías los cinco primeros lugares a nivel mundial (p.556).

Como se dijo, las manifestaciones de los trastornos mentales son variadas y complejas; van desde aquellos que evidencian un compromiso orgánico, hasta otros síntomas contemporáneos con una mayor influencia social como la depresión, la ansiedad, las adicciones, los intentos de suicidio y los trastornos de la conducta alimentaria, situaciones como dificultades relacionadas con la violencia, la sexualidad, el fracaso escolar o los duelos -que en psiquiatría son catalogadas como urgencias sociales- (Arango-Dávila et al., 2008; Murillo, Contreras \& Rincón, 2010; Moya et al., 2011; Belaga, 2006; Sotelo, 2007; Posada-Villa, 2009; Bulbena et al., 2007; Chinchilla \& Barbudo, 2010, p.643; Moya et al., 2011, p.25; Louro, Vásquez \& de la Cámara, 2011, p.1415).

Diferentes disciplinas con sus modelos explicativos intentan dar cuenta de estos fenómenos: la medicina en tanto ciencia basada en la evidencia (Moya et al., 2011. p.1), la enfermería, las teorías neurobiológicas y corrientes psicológicas de aspiración científica como la cognitivo-conductual (Serrano, 2012), además de otras orientaciones epistemológicas. En general puede afirmarse que cada uno de estos enfoques teóricos identifican factores biológicos, conductuales, emocionales, cognitivos, sociales y culturales que merecen ser tenidos en cuenta a la hora de proponer un plan terapéutico, pero ninguno de estos por sí solo explica completamente la naturaleza compleja de 


\section{Viana Ángela Bustos Arcón, Ana Rita Russo de Sánchez}

los llamados trastornos mentales, y por consiguiente diferentes disciplinas* están llamadas a responder (Arango-Dávila et al., 2008, p.554; Chinchilla, 2010; Berenzon \& Vargas, 2012, p.19).

En este sentido, la interdisciplinariedad representa un desafío sustancial para los diferentes profesionales a la hora de comunicarse y tomar decisiones que promuevan la cura del paciente o mitigación del impacto negativo de los trastornos en la vida de los sujetos que los padecen (Lyons, 2006; Chinchilla, 2010, p.4; Bulbena et al., 2007). Chinchilla (2010) lo ilustra al advertir que "el especialista tiende a diagnosticar aquello que sabe tratar y viceversa" (p.644), lo cual permite formular una pregunta en torno a cómo hacer un diálogo transdisciplinar y con qué alcance.

Según lo expuesto hasta ahora, es deducible por qué en el mundo la demanda de atención a la urgencia psiquiátrica resulta cada vez más frecuente (Cruz et al., 2007; Conde, Esteban, Rosado, Barroso \& Romero, 2009, p.303), tendencia que se ve reflejada también en Colombia. Posada-Villa (2009) comenta: "Se sabe que la prevalencia promedio de trastornos psiquiátricos en los servicios de urgencias es de un $24 \%$ de los pacientes atendidos, además de un $9 \%$ con sintomatología enmascarada" (p.217); el autor señala igualmente que en el país:

Es evidente que los servicios de urgencias han asumido, de manera progresiva, responsabilidades asistenciales de importancia en salud mental. En los últimos años, la atención de los casos psiquiátricos urgentes ha estado a cargo de los médicos generales y de otras especialidades de los servicios de urgencias y esto sigue siendo la regla. (Posada-Villa, 2009, p.217)

Esta afirmación indica que, bajo dichas condiciones, el personal asistencial de los servicios de urgencias de los hospitales generales deben tener la preparación para hacerle frente a las demandas de atención de esta naturaleza (Arango-Dávila et al., 2008, p.554; Chinchilla, 2010, p.6) y sumado al desafío de intervenir en salud mental, el contexto de la urgencia hace que el trabajo sea más complejo (Chinchilla, 2010).

En lo que respecta a las urgencias subjetivas, a pesar de que el concepto puede entenderse desde varios puntos de vista o tiene varias acepciones, en la teoría psicoanalítica este hace referencia a toda una teoría del sujeto, lo cual es conceptualmente diferente de la consideración psiquiátrica: en psicoanálisis se alude a la angustia sin anudar discursivamente, dejando significantes sueltos, por fuera del registro simbólico, generando una tensión psíquica insoportable, cuya manifestación es particular en cada paciente -por eso se formula en plural: 'urgencias subjetivas'-, en psiquiatría lo subjetivo en la urgencia apunta a una opinión del paciente sobre la gravedad de sus síntomas, un calificativo sin verificación clínica en el triage.

En ese sentido el concepto de "urgencias subjetivas" es tomado como referente teórico, pues consideramos que posibilita plantear estrategias que articulan los recursos disponibles en el hospital general y promover un diálogo de interfase entre las disciplinas implicadas en la atención urgente de los pacientes.

\section{MÉTODO}

Se realizó una revisión de la literatura de tipo descriptivo, desarrollada a través de un proceso de búsqueda de información, analizando documentos existentes en el metabuscador de Google 
Académico y en las bases de datos: Ebsco Host, PubMed, Scielo, Redalyc, Cuiden, y BVS, con criterios de inclusión de calidad: artículo de revistas de libre acceso al texto completo, revisiones sistemáticas de bases de datos y literatura impresa; los textos fueron elegidos por pertinencia, relevancia y accesibilidad, se tomaron como referencia los avances teóricos de investigadores como Belaga (2006), Sotelo (2007) y Seldes (2008).

\section{RESULTADOS}

\subsection{La urgencia médica}

En medicina no resulta fácil discriminar científicamente lo que es o no urgente porque esto involucra aspectos altamente subjetivos, así lo señalan Moya et al. (2011) cuando revelan que "no siempre coincide gravedad con urgencia y urgencia con gravedad" (p.26). Teniendo en cuenta esta proposición, la ciencia médica ha procurado hacer una distinción entre urgencia objetiva, también Ilamada urgencia real, y urgencia subjetiva (Murillo et al., 2010, p.353; Chinchilla \& Barbudo, 2010, p.639; Moya et al., 2011, p.6).

La urgencia médica verdadera u objetiva es definida como aquella situación donde necesariamente se requiere atención hospitalaria, que involucra un riesgo vital inminente o secuelas graves para el paciente según lo enuncian autores como Moya et al. (2011, p.5) y Chinchilla y Barbudo (2010, p.639). El concepto de urgencia subjetiva tiene lugar en medicina con la finalidad de dar crédito a la familia o el enfermo, ante una situación a la que se le concede un valor de gravedad y que desemboca en el pedido de intervención médica urgente (Murillo et al., 2010, p.353; Chinchilla \& Barbudo, 2010, p.639; Moya et al., 2011, p.6). Todos estos autores además exponen que la urgencia subjetiva con frecuencia es considerada o no como urgencia según el criterio del profesional de salud al aplicar los protocolos de valoración estandarizados en el sistema de salud.

Al igual que la urgencia médica, la definición rigurosa de la urgencia psiquiátrica evidencia tropiezos. Chinchilla (2010) indica que el concepto se encuentra poco delimitado objetivamente y que no existe un acuerdo en torno a su definición en la comunidad científica, lo que hace difícil su discriminación y tratamiento (p.4); además, autores como Cruz et al. (2007, p.109), Posada Villa (2009, p.218) y Ontiveros (2012, p.17) también han señalado que la ausencia de límites objetivables en su definición constituye un obstáculo importante para el personal asistencial en la atención de estos eventos.

\subsection{Urgencia psiquiátrica}

Para referirse a este concepto es inevitable remitirse a definiciones y procesos de las urgencias generales debido a que estas implican un modelo de organización asistencial similar (Ontiveros, 2012, p.217; Moya et al., 2011, p.41) y por la frecuencia con que se presentan problemas somáticos y psiquiátricos en el mismo sujeto (Chinchilla, 2010, p.3). Por consiguiente, Chinchilla (2010) explica que el abordaje de la urgencia psiquiátrica involucra situaciones más complejas que la urgencia general y muchas veces corre con el descrédito de que su atención sea considerada 
como aplazable. Es cierto que las urgencias medicoquirúrgicas implican un peligro real o potencial para la vida a causa de enfermedad, accidente o catástrofe, más la opinión del autor es que la urgencia psiquiátrica -además de estos riesgos y la aceleración del tiempo inherente-, evidencia los peligros propios que el paciente psiquiátrico puede sumar como consecuencia de sus trastornos comportamentales, secundarios a dificultades de adaptación o intolerancia al medio (p.4).

Bulbena et al. (2007) define la urgencia psiquiátrica como "aquella situación en la que unos síntomas psicopatológicos o un trastorno de conducta son percibidos como perturbadores o amenazantes por el propio paciente, de modo que llegan a producir una solicitud de atención psiquiátrica urgente" (p.2); teniendo en cuenta estas consideraciones se evidencia que la práctica profesional en este ámbito debe generar acciones basadas en la evaluación, manejo y toma de decisiones en el menor tiempo posible (Kaplan \& Sadock, 1996; Bulbena et al., 2007; Chinchilla, 2010; Murillo et al., 2010; Louro et al., 2011; Ontiveros, 2012).

Sin embargo, la finalidad de una evaluación psiquiátrica urgente en términos médicos es la valoración oportuna del paciente en crisis para decidir la prioridad de asistencia de acuerdo a su estado. Según Kaplan y Sadock (1996) lo más importante es determinar si el problema es orgánico, funcional o ambos, y por consiguiente no se espera que la evaluación de urgencias sea muy exhaustiva; como lo explica Teijeiro (2005) los esfuerzos están encaminados a disminuir el riesgo implícito en la situación detonante y no a darle una solución contundente al problema, que requiere de más tiempo. En virtud de esto, es clave el recurso a la interconsulta para aclarar o complementar la evaluación inicial, definir un diagnóstico y facilitar la elección del tratamiento (Kaplan \& Sadock, 1996, pp.8, 31).

Los manuales de psiquiatría sugieren que para hacer una valoración efectiva, el médico requiere habilidades para observar, escuchar y analizar (Kaplan \& Sadock, 1996, p.4; Chinchilla \& Barbudo, 2010, p.650; Murillo et al., 2010, p.346; Louro et al., 2011, p.1415; Ontiveros, 2012, p.217); además sugieren, entre otras intervenciones, la contención verbal, la psicoterapia, la contención física y la contención farmacológica, herramientas a disposición del equipo asistencial de urgencias para el manejo de situaciones difíciles con los pacientes cuya elección se encuentra sujeta a la formación "Psi"* del personal y su experiencia clínica con este tipo de pacientes.

\subsection{Urgencias subjetivas}

Este concepto propuesto por una vertiente del psicoanálisis lacaniano se diferencia en su significado del término en medicina, debido a que no se reduce exclusivamente a una percepción del paciente a la espera de ser validada por la ciencia, contrastándola con lo que se considera objetivamente verificable.

Para el psicoanálisis, en las urgencias subjetivas el interés clínico está centrado en la noción de sujeto, lo que no es igual a decir "individuo" o "yo" (Sotelo, 2007; Belaga, 2006; Seldes, 2006) porque se entiende que el surgimiento del sujeto, se da, no al momento de nacer, sino en el encuentro con el lenguaje que le preexiste (Lacan, 1960/1999, p.779); se hace referencia al sujeto del inconsciente. 


\section{Viana Ángela Bustos Arcón, Ana Rita Russo de Sánchez}

En ese sentido, la investigación psicoanalítica ha revelado cuestiones sobre el psiquismo humano que dan cuenta de su compleja estructuración subjetiva, más allá de la influencia genética, el desarrollo biológico y las manifestaciones de estos elementos sobre el comportamiento, para el psicoanálisis existen diferentes formas de constitución psíquica que resultan del encuentro del infante con el lenguaje: Estructuras clínicas conocidas como neurosis, psicosis y perversión. Las tres se organizan alrededor del complejo de castración (como le llamó Freud) y dependen de la inscripción del Nombre del Padre en términos de Lacan (Lacan, 1958/1999, p.183).

En relación con las estructuras clínicas, en psicoanálisis se parte de la idea de que al ser el sujeto un efecto del lenguaje hay efectos de pérdida de goce que se producen, afectando el cuerpo, la relación con la realidad y la relación con la ley, y por lo tanto la intervención está orientada fundamentalmente por el diagnóstico de estructura y no por la sintomatología que se exterioriza en el comportamiento del paciente (Laurent, 1989, p.114; Miller, 1987/1964, p.12). El diagnóstico de estructura resulta esencial porque la lectura de lo que le pasa al paciente tendrá unas características específicas y unas coordenadas de intervención radicalmente diferentes.

Sotelo (2007) realiza esta precisión: "mientras la medicina o el derecho van a buscar la objetividad en el diagnóstico, el movimiento que el psicoanálisis propone es ubicar la relación que tiene el sujeto con su padecimiento" (p.36).

El psicoanálisis considera que de una u otra forma la urgencia siempre es subjetiva, una experiencia singular, por eso habla de urgencias, y no de urgencia en un sentido universal. Belaga (2006) explica: "la lista es extensa: hay tantas urgencias como sujetos. Estos presentan a su vez, una constelación de fenómenos que los autores permiten reducir a dos fenómenos clínicos precisos. Dos que no engañan: la certeza y la angustia" (párr. 2). Lo urgente se exterioriza en tanto procede de una situación que para el sujeto no da espera, y aquello que no da espera suele manifestarse como angustia en sintonía con una vivencia traumática (Laurent, 2006; Belaga, 2006; Sotelo, 2007; Seldes, 2008).

Es posible afirmar que las lógicas del sufrimiento, la angustia y la crisis corresponden a la urgencia, constituyen el hilo conductor de la intervención, donde más allá del fenómeno que se exterioriza, lo que interesa es localizar el decir del paciente frente a lo que le sucede (Belaga, 2006, p.13; Laurent, 2006, p.29; Garmendía, 2006, p.49; Sotelo, 2006, p.105; Sotelo, 2007; Gorostiza, 2007; Seldes, 2008, párr. 5; Burgos, 2009). Según el psicoanálisis lo correcto clínicamente es favorecer que el sujeto ponga en palabras aquello que pasa por el cuerpo, intentando alojar en el discurso eso que se presenta desanudado (Belaga, 2006, p.17; Stiglitz, 2006, p.89; Sotelo, 2007; Seldes, 2008; Miller, 2009, p.255).

La intervención, implica de un lado al paciente, o sujeto que sufre, y del otro al terapeuta, o practicante como es nombrado por Gorostiza (2007). Consideramos necesario tomar en cuenta los cinco puntos que este autor trabaja al referirse a la dimensión formativa del terapeuta en la práctica con las urgencias subjetivas (Gorostiza, 2007, p.19):

1. "Es una práctica que potencia la exigencia de escuchar el detalle, el relieve singular en el discurso de quien consulta". En este punto el autor evidencia que la urgencia subjetiva exige un 


\section{Viana Ángela Bustos Arcón, Ana Rita Russo de Sánchez}

pasaje entre la clínica de la mirada a una clínica de la escucha (Foucault, 2004, p.157), es decir, no es suficiente la intervención a nivel del organismo pretendida por la medicina, el sujeto emerge cuando se le posibilita nombrar lo que le ocurre y encontrar vías para arreglárselas con eso.

2. "En tanto no se plantea responder desde un protocolo preestablecido, fuerza al practicante a ejercitarse en la disciplina de "olvidar lo que sabe" de antemano". Esto implica que la teoría se detiene ante al hecho del enfermo, la clínica es un ejercicio del saber, pero a la vez una producción de nuevo saber, por lo cual es necesario poner en suspenso las teorías y la imaginación y esperar que la observación de las cosas entreguen su sentido.

3. "Necesariamente está en juego la inventiva". Con esto Gorostiza refiere que las eventualidades de la urgencia fuerzan al practicante a ser recursivo, a encontrar en el aquí y el ahora vías para maniobrar con las contingencias reveladas por el paciente, sin olvidar que cada caso es diferente al anterior, y que la lógica que se pone en juego es la de la singularidad.

4. "Es una experiencia que lleva al practicante a enfrentarse con un equivalente de lo traumático constitutivo a todo hablante"; por lo tanto la actuación del terapeuta no se autoriza en un protocolo, sino en el texto subjetivo que entrega el paciente. En razón de esto hay una vía fundamental en la palabra, en términos de Miller (2009), "los criterios de curación en análisis son subjetivos, dependen de la palabra del sujeto" (p.255).

5. "Hace falta que se introduzca un tiempo en la urgencia, una pausa". El autor pone el acento aquí en la posibilidad de diferenciar el tiempo del sujeto del tiempo cronológico, basado en la enseñanza de Lacan (2000), donde explica que hay un instante para ver un tiempo para comprender y el momento de concluir (p.194). La pausa es el espacio necesario para incluir esta dimensión de la comprensión donde sea posible la transformación de las dificultades súbitas, o constantes, en algo diferente: construir una pregunta que lo implique responsablemente.

\section{DISCUSIÓN}

\subsection{Interfase Medicina - Clínica "Psi”}

Como se ha mencionado, la demanda de atención psiquiátrica urgente está tomando cada vez mayor fuerza, especialmente en los hospitales generales (Posada-Villa, 2009). De acuerdo con eso, es indispensable que el personal de los servicios de urgencias tenga una formación que responda a estas exigencias. La demanda de atención en los servicios de urgencias suelen caracterizarse por una aceleración del tiempo: se requiere una atención inmediata; esta es una relación al tiempo cronológico que por ninguna razón puede dejar de considerarse, más en las urgencias subjetivas se considera con mayor énfasis un tiempo lógico, aludiendo a la atemporalidad del inconsciente (Bialer, Dreyzin, Hornstein, Lifschitz, Manavella \& Michanié, 1988, p.12; Dreyzin \& Lichtensein, 1988, p.19). 


\section{Viana Ángela Bustos Arcón, Ana Rita Russo de Sánchez}

Ya se ha mencionado ampliamente que la clínica "Psi" evidencia que la escucha es un pilar fundamental de la intervención. Miller, citado por Sotelo (2007) propone para la clínica el término "avaluación", que implica "la evaluación con el aval del paciente" (p.35). Esto sugiere al clínico abstenerse de responder desde el lugar del saber, y conlleva a la implicación del sujeto en la búsqueda de salidas a lo que se exterioriza como urgente. Leguil (1988) en los comienzos de estos desarrollos de la clínica de la urgencia en el hospital ya evidenciaba marcadas diferencias entre la concepción del fenómeno en medicina y desde el campo "psi":

El médico concibe que la urgencia propulsa hacia "cosas por hacer"; nosotros vemos en cambio que en nuestro campo nos confronta con "cosas por decir" (...) Se trata aquí de coordenadas inversas: en su urgencia el médico selecciona las cosas por hacer y simplifica la situación, mientras que nosotros la tomamos compleja multiplicando las cosas por decir con nuestro compromiso, con nuestra introducción en la situación misma. (Leguil, 1988, pp.24-25)

Con esto se pretende introducir el hecho de que en las urgencias "psi", tanto la psiquiatría como el psicoanálisis tienen un aporte valioso que hacer, y en esa medida estas disciplinas, y otras tantas como la psicología, el trabajo social, la enfermería,... no se contraponen sino que pueden realizar un trabajo complementario, y por ello resulta útil pensar el tema de la interfase (Vaschetto, 2006; Levy, 2006; Stiglitz, 2006; Sotelo, 2007), como un trabajo que hace referencia a la intervención interdisciplinaria.

Vaschetto (2006) lo propone así: "podemos pensar la intersección psiquiatría-psicoanálisis como una operación constitutiva y estructural donde tanto la psiquiatría como el psicoanálisis superponen sus carencias en tanto respuestas a lo real del sufrimiento" (p.166). Del lado de la psiquiatría, Arango-Dávila et al. (2008) lo ilustran en la siguiente afirmación: "Algunas condiciones psiquiátricas responden mejor al manejo con estrategias biológicas, otras muestran mejores resultados con la psicoterapia, mientras que entre una y otra están muchos trastornos que pueden ser manejados con combinaciones de uno y otro enfoque" (p.558).

\subsection{Manuales diagnósticos, psicofarmacología y la palabra}

En la práctica de la evaluación psiquiátrica urgente es notable que los equipos asistenciales hayan centrado su interés en apuntar a un diagnóstico basado en los manuales de clasificación diagnóstica DSM y CIE, para luego proceder ágilmente según las guías de atención médica. Aunque entre las intervenciones en psiquiatría se pueden encontrar la contención verbal, la contención física, la contención farmacológica, la psicoterapia, entre otras, es muy frecuente en la práctica médica el recurso al medicamento como vía privilegiada para intervenir (Lichtensztajn, 1988, p.47; Vaschetto, 2006, p.167), lo que ha llevado a silenciar el saber del sujeto, y la posibilidad de asumirse como éticamente responsable al preguntarse por lo que le sucede (Garmendía, 2006, p.50).

Laurent (1989) señala que la actual clínica "psi" está en favor de "continuos sindrómicos reordenados por la clínica del medicamento" (p.114); asunto del que también se han ocupado otros autores que denuncian el abuso en la prescripción de psicofármacos al paciente que demanda atención en este campo (Kuperman, 1988; Gallano, 1995; Uzorskis, 1995; Burgos, 2009). 


\section{Viana Ángela Bustos Arcón, Ana Rita Russo de Sánchez}

Siendo consecuentes con lo planteado hasta ahora, abordando estos problemas desde el marco de las urgencias subjetivas, se busca responder desde otro ángulo a la crisis que enfrentan los manuales y la atención protocolizada en medicina, entendiendo que los motivos que determinan una consulta de urgencia "psi" exceden el marco de las categorías psicopatológicas (Levy, 2006, p.120; Belaga, 2006, p.13; Vaschetto, 2006, p.168).

Indudablemente la medicación es un recurso que puede prestar gran utilidad para el manejo del paciente en crisis, allí donde no hay posibilidad de alojar la palabra para escuchar el aspecto simbólico del síntoma (Ivanier, 1988, p.74). En opinión de Ivanier (1988), “la indicación de psicofármacos apuntará a reducción del goce y la restitución del paciente a su condición de sujeto, al permitirle de nuevo su expresión por la palabra" (p.74). Esto resulta de gran importancia porque implica reconocer los límites del modelo médico y los límites de la intervención en un dispositivo de palabra; así lo ilustra Stiglitz (2006) cuando afirma: "como el medicamento el sentido tiene efectos terapéuticos y efectos iatrogénicos, por lo que su uso debe ser prudente" (p.85).

Se busca situar que tanto los manuales diagnósticos y la psicofarmacología, como la palabra, son herramientas de las que disponen los equipos asistenciales en las instituciones hospitalarias; lo que resulta fundamental es que en el abordaje de la urgencia psiquiátrica se realice un análisis clínico que trascienda la presentación superficial de los síntomas y tenga en cuenta al sujeto del inconsciente, reconociendo que la superación de la crisis por medio de una intervención del cuerpo biológico no es suficiente. De igual manera, el tratamiento por el sentido no siempre lo será "cuando aceptamos la medicación o el ingreso admitimos que hablar y ser escuchado sigue siendo necesario pero no suficiente" (Garmendía, 2006, p.53).

De esta manera se pretende enunciar que es viable, y además necesario, alojar en la atención de la urgencia psiquiátrica una clínica de la escucha que permita al sujeto revelarse pues "la escucha en la urgencia puede orientar el caso hacia un posible tratamiento, puede introducir algún interrogante para el sujeto que le implique posteriormente en aquello que le ha sucedido" (Garmendía, 2006, p.44). Al afirmar esto, no se pretende "forzar a los médicos a tolerar algo para lo cual se sienten cada vez menos preparados (...) sin pretender hacer de ellos analistas, ni aliviarlos de su responsabilidad" (Gamsie, 1995); pero es claro que si no hay sujetos del lado del paciente, únicamente quedarán usuarios o clientes, en una frívola relación con un técnico que actúa esquemáticamente, despojado de toda su habilidad como terapeuta (Burgos, 2009, p.24).

\subsection{Hospital general y urgencia psiquiátrica}

Las normas de acreditación de los sistemas de calidad actualmente exigen a las instituciones organizar su obrar, creando dispositivos y protocolos de intervención para tratar las dificultades de los pacientes; si bien esto puede ser entendido como esquematizante y rígido, las instituciones pueden apoyarse en tales esquemas en un sentido general para tener un punto de apoyo, pues será necesario que existan criterios orientadores para la toma de decisiones (Levy, 2006, p.117; Vaschetto, 2006, p.167). Ahora bien, teniendo en cuenta el concepto de urgencias subjetivas, podría pensarse que existe una exclusión radical del término en el marco de los hospitales generales; no obstante, hay una oportunidad para no "pelearse" con los protocolos si se considera 


\section{Viana Ángela Bustos Arcón, Ana Rita Russo de Sánchez}

que la práctica clínica, como bien lo enuncia Levy (2006), "se desprende de un conocimiento y de una ética" (p.115) que permiten un margen de maniobra que involucra la lógica del caso por caso.

En este sentido, en el marco de la atención hospitalaria a pacientes que consultan por una urgencia "psi", el manejo de las entrevistas con los sujetos cobra un gran interés, permitiendo que sean algo más que un espacio para llenar un formato; es necesario considerar seriamente su contenido significante y dentro de esto es decisiva la posición subjetiva del profesional que está en interacción con esa persona que sufre, alojando en una escucha respetuosa lo que el otro tiene por decir (Levy, 2006, p.119 ; Vaschetto, 2006, p.167; Sotelo, 2007, p.30); el psicoanálisis enseña mucho sobre la diferencia de un abordaje objetivizante que silencia al sujeto y uno subjetivizante que le supone un saber, abriendo un espacio para interrogarse sobre lo que le pasa, por lo tanto en este punto es fundamental recurrir a la interconsulta y el trabajo interdisciplinar.

Al respecto, Sotelo (2007) "destaca la importancia de reunirse en la diversidad de formación, en la diversidad de tareas, en la diversidad ideológica, y tratar de construir el espacio institucional como espacio creativo (...) Buscar un hilo en común" (p.25), lo que pone el acento en la importancia de reconocer que en salud mental hay un importante entrecruzamiento de distintos campos del saber. No obstante, la autora también señala la dificultad de realizar un diálogo interprofesional cuando existen marcadas diferencias entre lo que se considera normal y patológico (Sotelo, 2007, p.29).

La normatividad colombiana exige a las entidades prestadoras de servicios de salud cubrir la atención en salud mental (Ministerio de Salud y Protección Social, 2013; Ministerio de Salud y Protección Social, 2013a), atendiendo al derecho constitucional a la salud integral. Los hospitales generales, ya sean de primer nivel o de mayor complejidad, tienen una oportunidad en la reciente normatividad en salud mental (Ley 1616, 2013) para abordar las urgencias "psi" con mayor eficacia. La atención a la urgencia psiquiátrica teniendo como centro la noción de sujeto que ha aportado el psicoanálisis, implica que el personal médico y de enfermería no pasen por alto la existencia de las determinaciones inconscientes de sus pacientes, y en ese sentido, tengan claridad de que además de atender el cuerpo, es fundamental abrir otra vía en los dispositivos de palabra mediante la interconsulta (Canedo, 2009, p.29; Ayala \& Solís, 2012, párr. 4).

Retomando las palabras de Kuperman (1988), se sintetiza en una frase parte de todo lo dicho hasta ahora: "El recetario del hospital puede servir también para prescribir 'venir a hablar'” (p.78).

Nota de Autores

Artículo derivado de la investigación: Atención eficaz al paciente que consulta por una urgencia psiquiátrica en el servicio de urgencias de un hospital general de tercer nivel de la ciudad de Bogotá. 


\section{REFERENCIAS}

Arango-Dávila, C., Rojas, J. C. \& Moreno, M. (2008). Análisis de los aspectos asociados a la enfermedad mental en Colombia y la formación en psiquiatría. Revista Colombiana de Psiquiatría, 37(4), 538563. Recuperado de: http://www.redalyc.org/articulo.oa?id=80637406

Ayala, C. \& Solís, C. (2012). Otras posibles aplicaciones clínicas de fármacos con efecto 5HT2A y 3 en psiquiatría de enlace: reporte de casos. Revista Colombiana de Psiquiatría, 40(1), 217-229. Doi: 10.1016/ S0034-7450(14)60079-2 http://www.redalyc.org/articulo.oa?id=80624093016

Belaga, G. (2006). Presentación. En G. Belaga, La urgencia generalizada: La práctica en el hospital (pp. 1119). Buenos Aires, Argentina: Grama Ediciones.

Berenzon, S. \& Vargas, I. (2012). Salud mental y sociedad. En J. R. de la Fuente \& G. Heinze (Ed.), Salud mental y medicina psicológica (pp. 12-21). Mé- xico: McGraw-Hill. Recuperado de : http://totorus.tic.unam.mx/9912/pdf/99delafuente.pdf

Bialer, M., Dreyzin, A., Hornstein, C., Lifschitz, G., Manavella, N. \& Michanié, L. (1988). La urgencia. En V. autores, La urgencia: El psicoanalista en la práctica hospitalaria. Buenos Aires: Ricardo Vergara Ediciones.

Bulbena, A., Martin, L. \& Arcega, J. (2007). Experiencia de una Unidad Móvil de Urgencias Psiquiátricas en Barcelona. Avances en Salud Mental Relacional, 6(3), 1-14. https://www.researchgate.net/profile/Antonio_Bulbena3/publication/237828891_EXPERIENCIA_ DE_UNA_UNIDAD_MOVIL_DE_URGENCIAS_PSIQUIATRICAS_EN_BARCELONA_EMSE-

061_Equipo_Multidisciplinar_de_Soporte_Especializado/links/02bfe511b8481b6716000000/EXPE RIENCIA-DE-UNA-UNIDAD-MOVIL-DE-URGENCIAS-PSIQUIATRICAS-EN-BARCELONA-EMSE-061Equipo-Multidisciplinar-de-Soporte-Especializado.pdf

Burgos, F. (2009). ¿Para qué diagnosticamos? L'interrogant, (9), 20-24. Canedo, M. (2009). Psiquiatría de interconsulta y enlace. Galicia Clin, 1(70), 29-30.

Chinchilla, A. (2010). Conceptos y peculiaridades de las urgencias psiquiátricas. En A. Chinchilla, J. Correas, F. Quintero \& M. Vega (Eds.), Manual de urgencias psiquiátricas (segunda ed., pp. 3-16). Barcelona: Elsevier.

Chinchilla, A. \& Barbudo, E. (2010). Uso y abuso de la urgencia psiquiátrica. En A. Chinchilla, J. Correas, F. Quintero \& M. Vega (Eds.), Manual de urgencias psiquiátricas (segunda ed., pp. 639-652). Barcelona: Elsevier.

Conde, M., Esteban, C., Rosado, L., Barroso, M. \& Romero, S. (2009). La urgencia psiquiátrica en un hospital general. La patología de la agresividad principal motivo de consulta. Revista Asociación española de psiquiatría, 29(104), 303-317. Recuperado de : http://scielo.isciii.es/scielo.php?script=sci_arttext\&pid=S0211-57352009000200002

Cruz, C., Leiva, J., Escobar, E., Eugenín, D., Errázuriz, M., Prieto, B. \& Rinaldi, S. (2007). Demanda espontánea de urgencias psiquiátricas al Hospital Asistencia Pública Dr. Alejandro del Río. Revista 


\section{Viana Ángela Bustos Arcón, Ana Rita Russo de Sánchez}

Chilena de Neuro-psiquiatría, 45(2), 108-113. DOI: http://dx.doi.org/10.4067/S071792272007000200003

Dreyzin, A. \& Lichtenstein, A. (1988). Eric Laurent con el grupo de investigación sobre las urgencias. En V. autores, La urgencia: El psicoanalista en la práctica hospitalaria. Buenos Aires: Ricardo Vergara Ediciones.

Foucault, M. (2004). El nacimiento de la clínica: Una arqueología a la mirada médica. Buenos Aires: Siglo $X X I$.

https://books.google.es/books?hl=es\&lr=\&id=8k2ef8FjNXAC\&oi=fnd\&pg=PA1\&dq=+El+nacimiento+de+la+ cl\%C3\%ADnica:+Una+arqueolog\%C3\%ADa+a+la+mirada+m\%C3\%A9dica\&ots=vhxQ39yE9k\&sig=ziT B-Q7pym3Yp0-

https://books.google.es/books?hl=es\&lr=\&id=8k2ef8FjNXAC\&oi=fnd\&pg=PA1\&dq=+El+nacimiento + de+la+cl\%C3\%ADnica:+Una+arqueolog\%C3\%ADa+a+la+mirada+m\%C3\%A9dica\&ots=vhxQ39yE9k \&sig=ziTB-Q7pym3Yp0-

sRM8hLxhHfGA\#v=onepage\&q=El\%20nacimiento\%20de\%20la\%20cl\%C3\%ADnica\%3A\%20Una\%20 arqueolog\%C3\%ADa\%20a\%20la\%20mirada\%20m\%C3\%A9dica\&f=falsesRM8hLxhHfGA\#v=onepage \&q=El\%20nacimiento\%20de\%20la\%20cl\%C3\%ADnica\%3A\%20Una\%20arqueolog\%C3\%ADa\%20a\% 20la\%20mirada\%20m\%C3\%A9dica\&f=false

Gallano, C. (1995). La salud mental y la clínica del sujeto. Psicoanálisis y el hospital, 4(7), 8-10.

Garmendía, J. (2006). Urgencia psiquiátrica: Una perspectiva psicoanalítica. En G. Belaga, La urgencia generalizada: La práctica en el hospital (pp. 43-71). Buenos Aires: Grama Ediciones. Recuperado de http://nucep.com/wp-content/uploads/2013/01/Javier-Garmendia-_URGENCIA-PSIQUIATRICA.UNA-PERSPECTIVA-PSICOANALITICA.pdf

Gorostiza, L. (2007). Prólogo. En I. Sotelo, Clínica de la urgencia (pp. 15-20). Buenos Aires: JCE Ediciones.

Ivanier, M. (1988). A cerca del lugar de los psicofármacos en la urgencia. En Varios, La urgencia: El psicoanalista en la práctica hospitalaria (pp. 73-75). Buenos Aires: Ricardo Vergara Ediciones.

Kaplan, H. \& Sadock, B. (1996). Psiquiatría de Urgencias: Introducción. En H. I. Kaplan \& B. J. Sadock, Manual de psiquiatría de urgencias (pp. 3-28). Buenos Aires: Panamericana.

Kuperman, M. (1988). Clopromazina: Marca registrada para el sujeto. En Varios, La urgencia: El psicoanalista en la práctica hospitalaria (pp. 77-79). Buenos Aires: Ricardo Vergara Ediciones.

Lacan, J. (1958-1999). La lógica de la castración. En Seminario 5 Las formaciones del inconsciente (pp. 145256). Argentina: Paidós. Rescatado de: https://es.scribd.com/document/355363118/El-Seminario5-Las-formaciones-del-inconsciente-Jacques-Lacan-pdf

Lacan, J. (1960-1999). Subversión del sujeto y la dialéctica del deseo en el inconsciente freudiano. En Escritos 2 (pp. 773-807). Argentina: Siglo XXI. 
Lacan, J. (2000). El tiempo lógico y el acerto de certidumbre anticipada: Un nuevo sofisma. En Escritos 1 (pp. 187-203). Argentina: Paidós. Recuperado de: https://es.scribd.com/document/341170540/Lacan-El-tiempo-logico-y-el-aserto-de-certidumbreanticipada-pdf

Laurent, E. (1989). Melancolía, dolor de existir, cobardía moral. En E. Laurent, Estabilizaciones en las psicosis (pp. 113-125). Argentina: Manantial.

Laurent, E. (2006). Hijos del trauma. En G. Belaga, La urgencia generalizada: La práctica en el hospital (pp. 23-29). Buenos Aires: Grama Ediciones. Recuperado de: https://es.scribd.com/doc/297514646/Laurent-E-2004-Hijos-Del-Trauma-en-La-UrgenciaGeneralizada-La-Practica-en-El-Hospital-Pp-23-29-Editorial-Grama

Leguil, F. (1988). Reflexiones sobre la urgencia. En Varios, La urgencia: El psicoanalista en la práctica hospitalaria (pp. 23-28). Buenos Aires: Ricardo Vergara Ediciones.

Levy, E. (2006). Sobre el manejo de la urgencia. En G. Belaga, La urgencia generalizada: La práctica en el hospital (pp. 115-128). Buenos Aires: Grama Ediciones+

Lichtensztajn, J. (1988). La urgencia, la escanción. En Varios, La urgencia: El psicoanalista en la urgencia hospitalaria (pp. 47-49). Buenos Aires: Ricardo Vergara Ediciones.

Louro, J., Vásquez, C. \& de la Cámara, M. (2011). Urgencias psiquiátricas. En M. Moya, P. Piñera \& M. Mariné, Tratado de medicina de urgencias (Vol. II, pp. 1415-1437). Madrid: Ergon. Recuperado de: http://www.scielo.org.co/pdf/psico/v21n39/0124-0137-psico-21-39-00203.pdf

Lyons, J. (2006). La complejidad de la comunicación en un entorno con múltiples disciplinas y profesionales: comunicometría y apoyo a la decisión. Medical Clinics of North America, 90(4), 693701. Doi: http://dx.doi.org/10.1016/j.mcna.2006.05.004

Miller, J.A. (1987-1964). Acción de la estructura. En Matemas I (pp. 6-23). Buenos Aires: Manantial.

Miller, J.A. (2009). La ética del psicoanálisis (1989). En J.-A. Miller, Conferencias Porteñas (Vol. I, pp. 253267). Argentina: Paidós.

Ministerio de Salud y Protección Social de Colombia. (21 de enero de 2013). Ley de Salud Mental. [Ley 1616 de 2013] Doi: http://www.descentralizadrogas.gov.co//pdfs/politicas/nacionales/ Ley_1616_de_2013.pdf

Ministerio de Salud y Protección Social de Colombia. (27 de diciembre de 2013a). Resolución Plan Obligatorio de Salud (POS). [Resolución 5521]. Doi: https://www.minsalud.gov.co/sites/rid/Lists/ BibliotecaDigital/RIDE/DE/DIJ/resolucion5521-de-2013.pdf

Moya, M., Piñera, P. \& Mariné, M. (2011). Bases conceptuales y organizativas de la medicina de urgencias. En M. Moya, P. Piñera \& M. Mariné, Tratado de medicina de urgencias (Vol. I, pp. 1-41). Madrid: Ergon. Recuperado de: https://es.scribd.com/document/280866394/Bases-conceptuales-yorganizativas-de-la-Medicina-de-Urgencias-pdf 
Murillo, N., Contreras, E. \& Rincón, H. (2010). Urgencias en psiquiatría. En L. Quintero (Ed.), Situaciones críticas en medicina de urgencias (pp. 345-354). Cali: Feriva.

Ontiveros, M. (2012). Urgencias en Psiquiatría. En J.R. De la Fuente \& G. Heinze (Ed.), Salud mental y medicina psicológica (pp. 216-229). México: McGrawHill.

Posada-Villa, J. (2009). Urgencias psiquiátricas. En Ministerio de Salud y Protección Social (Ed.), Guías para manejo de urgencias (Tercera ed., Vol. III, pp. 217-221). Bogotá: Editorial Ministerio de Salud y Protección Social. Recuperado de: https://www.minsalud.gov.co/Documentos\%20y\%20Publicaciones/Gu\%C3\%ADas\%20para\%20ma nejo\%20de\%20urgencias\%20-Tomo\%20III.pdf

Seldes, R. (2006). La urgencia subjetiva: un nuevo tiempo. En G. Belaga, La urgencia generalizada: La práctica en el hospital (pp. 31-42). Buenos Aires: Grama ediciones.

Seldes, R. (2008). La urgencia del psicoanálisis aplicado. Recuperado de http://virtualia.eol.org.ar/018/pdf/ seminario_seldes.pdf

Serrano, C. (2012). Tratamientos psicoterapéuticos. En J.R. De la Fuente \& G. Heinze (Ed.), Salud mental y medicina psicológica (pp. 286-297). México: McGraw-Hill.

Sotelo, I. (2006). La guardia, la admisión, la primera consulta: una coyuntura de emergencia. En G. Belaga, La urgencia generalizada: La práctica en el hospital (pp. 97-113). Buenos Aires: Grama Ediciones.

Sotelo, I. (2007). Clínica de la Urgencia. Buenos Aires, Argentina: JCE Ediciones.

Stiglitz, G. (2006). Inventar la lengua que se habla: Sobre eficacia y psicoanálisis. En G. Belaga, La urgencia generalizada: La práctica en el hospital (pp. 85-93). Buenos Aires: Grama Ediciones. Teijeiro, R. (2005). Triaje en la psiquiatría de urgencias. [En linea] Revista Psiquiatría, 9(2) [fecha de consulta: 21 de enero de 2013]. Recuperado de: .http://www.psiquiatria.com/psiq_de_enlace/triage-en-lapsiquiatria-de-urgencias/\#

Uzorskis, B. (1995). La clínica psicoanalítica en territorio médico. Psicoanálisis y el hospital, 4(7), 64-68.

Vaschetto, E. (2006). Urgencias de la salud mental. En G. Belaga, La urgencia generalizada: La práctica en el hospital (pp. 163-169). Buenos Aires: Grama Ediciones.

WHO (2011). Mental health: Mental Health Atlas 2011. Recuperado de http://www.who.int/mental_health/publications/mental_health_atlas_2011/en/ 